\title{
Curcumin Loaded Microsponges for Colon Targeting in Inflammatory Bowel Disease: Fabrication, Optimization, and In Vitro and Pharmacodynamic Evaluation
}

\author{
Rashmi Sareen, Kavita Nath, Nitin Jain, and K. L. Dhar \\ School of Pharmaceutical Sciences, Shoolini University, Bajhol, Solan, Himachal Pradesh 173229, India \\ Correspondence should be addressed to Rashmi Sareen; sareenrashmi@gmail.com
}

Received 5 February 2014; Revised 10 May 2014; Accepted 28 May 2014; Published 1 July 2014

Academic Editor: Florence Siepmann

Copyright (c) 2014 Rashmi Sareen et al. This is an open access article distributed under the Creative Commons Attribution License, which permits unrestricted use, distribution, and reproduction in any medium, provided the original work is properly cited.

\begin{abstract}
The present study was aimed to develop and optimize the microsponges of curcumin for colon specific drug delivery in a view to bypass the upper gastrointestinal tract (GIT) for enhanced therapeutic effect. Microsponges were developed by quasi emulsion solvent diffusion method using $3^{2}$ full factorial design. Prepared microsponges were optimized in order to analyze the effects of independent variables (volume of ethanol and Eudragit L100) on the encapsulation efficiency, particle size, and drug release. The optimized formulation was subjected to in vivo study using acetic acid induced colitis model in rats. The F7 was selected as optimized formulation based on particle size of $41.63 \mu \mathrm{m}$, \% entrapment efficiency of $78.13 \%$, and \% cumulative drug release of $84.12 \%$, and desirability factor of 0.83 . Release studies revealed that microsponges prevented the premature release of curcumin in upper GIT and specifically released the drug at colonic $\mathrm{pH}$. The drug release profile of F7 formulation was subjected to different kinetic models and based upon the best correlation coefficient $\left(r^{2}=0.9927\right)$ the release was found to follow Higuchi model, which suggested diffusion as the main mechanism of drug release. Pharmacodynamic study showed that curcumin loaded microsponges causes a significant decrease in edema, necrosis, and hemorrhage of colon as compared to free curcumin. This study proves that curcumin loaded microsponges may act as a promising drug delivery system for treatment of ulcerative colitis.
\end{abstract}

\section{Introduction}

Inflammatory bowel diseases (IBDs) are idiopathic inflammatory and relapsing disorders of the digestive tract. Ulcerative colitis (UC) and Crohn's disease are the two major chronic IBDs involving the large intestine or colon $[1,2]$. Conventional drug therapy to treat IBD mainly includes the use of anti-inflammatory drugs which encounters obstacle such as absorption and degradation in the upper GIT and also causes serious side effects in upper GIT [3]. Thus, in the last decade colon-targeted drug delivery systems have gained significant interest due to their well-documented advantages such as specific drug targeting, reduction of doses and systemic side effects, and enhanced drug efficacy [4]. Microsponge delivery system (MDS) is the unique technique which provides targeted and controlled release of drug. Another added advantage of microsponges which contributes to their use is the ability of retaining drug on the surface of colon ensuring local and targeted action. Consequently, the lag time for drug absorption increases which makes MDS suitable for colon specific delivery [5]. MDS is highly porous, polymer based, cross linked, spongy spheres that have the ability to entrap and adsorb a wide range of active ingredients like anti-inflammatory, antifungal, antimicrobial, emollient, essential oils, and so forth. Microsponges have particle size ranging from 5 to $150 \mu \mathrm{m}$ and are highly efficacious, stable, nonirritant, nontoxic, nonallergic, nonmutagenic. It also reduces side effects and increases elegance and is compatible with the active ingredients [6]. Curcumin is a yellow-colored naturally occurring polyphenol obtained from the rhizomes of Curcuma longa. The safety and pharmacological efficacy of curcumin make it a suitable molecule for the prevention and treatment of various diseases. It is a highly pleiotropic molecule which can interact with different molecular targets involved in inflammation [7]. Curcumin also acts effectively against ulcerative colitis. It can modulate inflammatory 
TABLE 1: Disadvantages of various curcumin colon targeted formulations.

\begin{tabular}{ll}
\hline Formulation & Disadvantage \\
\hline Guar-gum based tablet & $\begin{array}{l}\text { Single unit system has disadvantage of unintentional disintegration of } \\
\text { tablet in GIT, which may lead to compromised systemic bioavailability. }\end{array}$ \\
\hline Microspheres and nanoparticles & $\begin{array}{l}\text { Microspheres and nanoparticles are comparatively nonporous in nature } \\
\text { and have less capability to bind to the rough surface of intestinal mucosa as } \\
\text { compared to microsponges particles. }\end{array}$ \\
\hline
\end{tabular}

response by downregulating the activity of cyclooxegenase2 [COX-2], lipoxygenase, and inducible iNOS [nitric oxide synthase] enzyme $[8,9]$. Curcumin is of natural origin and hence safe and nontoxic even at higher doses [10]. In last decade various colon targeting formulations of curcumin have been suggested by several researchers such as guar gum based tablets, microspheres, nanoparticles, and so forth. But these formulations have some drawbacks, which can be overcome by formulating microsponges as shown in Table 1. In this study we aimed at fabrication of curcumin loaded microsponges for colonic drug delivery. Curcumin loaded microsponges were prepared by quasi emulsion solvent diffusion method using Eudragit and water soluble porogen. Microsponges were optimized using $3^{2}$ full factorial design. The effect of independent variables on the particle size, entrapment efficiency, and \% cumulative drug release was evaluated. Finally, the optimized formulation was subjected to in vivo study using acetic acid induced colitis model in rats.

\section{Material and Methods}

2.1. Materials. Curcumin was obtained as a gift sample from Konark Herbal and Health Care, Mumbai, India. Eudragit L100 was received from Degussa India Pvt. Ltd., and polyvinyl alcohol (PVA) was purchased from the Nice Laboratory Reagent. Other excipients used were of standard pharmaceutical grade and all chemicals and reagents used were of analytical grade.

2.2. Fabrication of Microsponges. Microsponges were prepared by quasi emulsion technique using porogen. A (1\% aqueous solution of sodium chloride) was prepared which was used as porogen. A sufficient amount of Span 80 was added to the prepared porogen with agitation to obtain $1 \%$ (v/v) dispersion. A solution of curcumin and Eudragit L100 [11] was prepared in ethanol. Then porogen solution was uniformly emulsified in curcumin and Eudragit solution. $5 \% \mathrm{w} / \mathrm{v}$ aqueous PVA solution was prepared separately. Then the earlier prepared dispersion was emulsified in PVA solution. This emulsion was continuously stirred for $3 \mathrm{~h}$ to get microsponges. Then the microsponges were filtered and dried at $60^{\circ} \mathrm{C}$ and stored in desiccators [12]. Microsponges were prepared based on $3^{2}$ full factorial design and total nine formulations were prepared [F1-F9]. Each formulation varied by volume of ethanol and amount of Eudragit at three different levels (Table 2).
TABLE 2: $3^{2}$ factorial design for preparing curcumin loaded microsponges.

\begin{tabular}{lcccc}
\hline Formulations & $\begin{array}{c}\text { Drug } \\
(\mathrm{mg})\end{array}$ & $\begin{array}{c}\text { PVA } \\
(\mathrm{g})\end{array}$ & $\begin{array}{c}\text { Eudragit } \\
(\mathrm{mg})\end{array}$ & $\begin{array}{c}\text { Ethanol } \\
(\mathrm{mL})\end{array}$ \\
\hline F1 & 800 & 5 & 400 & 5 \\
F2 & 800 & 5 & 600 & 5 \\
F3 & 800 & 5 & 800 & 5 \\
F4 & 800 & 5 & 400 & 6 \\
F5 & 800 & 5 & 600 & 6 \\
F6 & 800 & 5 & 800 & 6 \\
F7 & 800 & 5 & 400 & 7 \\
F8 & 800 & 5 & 600 & 7 \\
F9 & 800 & 5 & 800 & 7 \\
F10* & 800 & 5 & 700 & 6.5 \\
\hline
\end{tabular}

${ }^{*}$ Extra design checkpoint.

\subsection{In Vitro Characterization of Microsponges}

2.3.1. Determination of Particle Size, Shape, and Surface Morphology. The curcumin loaded microsponges formulations were analyzed for determination of average diameter by ZetaSizer (Malvern Instruments, Mastersizer 2000, UK). The values $\left(d_{50}\right)$ were denoted for all formulations as average size range [13].

The microsponge formulations were visualized by scanning electron microscope (SEM) to assess the morphology of the microsponges and surface. Samples were coated with gold-palladium under an argon atmosphere at room temperature and the morphology of the microsponges was studied with SEM at $10 \mathrm{kV}$ (QUANTA 250, FEI Makers, Singapore) [14].

\subsubsection{Determination of Encapsulation Efficiency, Percentage} Yield, and Drug Loading. Curcumin microsponges [100 mg] were crushed and extracted using $10 \mathrm{~mL}$ methanol by vortexing and centrifuging at $2000 \mathrm{rpm}$ for $10 \mathrm{~min}$. Then insoluble residue was separated and the supernatant was analyzed spectrophotometrically at $430 \mathrm{~nm}$ after appropriate dilution. Then, the encapsulation efficiency, percentage yield, and drug loading were calculated by the following equation [15]:

Encapsulation efficiency (EE)

$$
=\left(\frac{\text { Mass of drug in microsponges }}{\text { Initial mass of drug }}\right) \times 100 \text {, }
$$


Percentage yield (PY)

$$
\begin{aligned}
& =\left(\frac{\text { Mass of obtained microsponges }}{\text { Initial mass of drug }+ \text { Initial mass of polymer }}\right) \\
& \quad \times 100 \text {, }
\end{aligned}
$$

Drug loading (DL)

$$
=\left(\frac{\text { Mass of drug in microsponges }}{\text { Mass of microsponges }}\right) \times 100 \text {. }
$$

2.4. In Vitro Drug Release. Dissolution study of curcumin microsponges was carried out in USP dissolution test apparatus II stirred at $100 \mathrm{rpm}$ and temperature of $37 \pm 0.5^{\circ} \mathrm{C}$ (Paddle type, Electro lab. EDT-08 11LX). Drug release was monitored for $12 \mathrm{~h}$ and samples were withdrawn periodically and sink conditions were maintained by replacing with equal amount of fresh dissolution medium. The dissolution was carried out at different $\mathrm{pH}$ condition using $0.1 \mathrm{~N} \mathrm{HCl}(\mathrm{pH} 1.2)$ for $2 \mathrm{~h}$, phthalate buffer ( $\mathrm{pH} 4.5$ ) for next $2 \mathrm{~h}$, and phosphate buffer ( $\mathrm{pH}$ 6.8) with $4 \% \mathrm{w} / \mathrm{v}$ fresh rat caecal content (RCC) under anaerobic condition for subsequent hours to simulate the GIT condition. After $12 \mathrm{~h}$ study, the samples were analyzed by UV spectrophotometer at $430 \mathrm{~nm}[16,17]$.

2.5. Optimization of Experimental Design. The effect of independent variables (volume of ethanol and Eudragit L100) on the dependent variables (\% EE, \% cumulative drug release, particle size) was modeled by Design Expert software version 8.0.7.1 [18]. Further theoretical formulation F10 was designed as an extra checkpoint to validate the predictability of optimization study (Table 2).

\subsection{In Vivo Study}

2.6.1. Acetic Acid Induced Experimental Ulcerative Colitis in Colon. Wistar Albino rats (body weight $=160-200 \mathrm{~g}$ ), $n=5$, were selected and were caged individually with food and water ad libitum. All the studies were conducted with prior approval of Institutional Animal Ethical Committee (IAEC/SU-PHARM/13/006).

The rats were distributed randomly in three groups, that is, control, curcumin, and microsponges of curcumin, each comprising five animals. $1 \mathrm{~mL}(4 \%)(\mathrm{v} / \mathrm{v})$ of acetic acid was given to induce ulcerative colitis in rats through intrarectal route which resembled with the inflammatory bowel disease in all groups $[19,20]$. For three days, rats were housed without any treatment to maintain the development of full IBD model. Each group received the treatment orally in $1 \%$ carboxymethyl cellulose (w/v) solution. Group 1 received vehicle only, group 2 received pure curcumin $(20 \mathrm{mg} / \mathrm{kg})$, and group 3 received microsponges of curcumin $(20 \mathrm{mg} / \mathrm{kg})$ $[21,22]$.

2.6.2. Pharmacological Assessment. After $24 \mathrm{~h}$ of last drug administration, the animals were sacrificed, colon part was cut, and ulcer projections were visualized and assessed on the basis of the inflammatory scales; that is, $0=$ normal colored colon, $0.5=$ red coloration, $1=$ spot ulcer, $1.5=$ hemorrhagic streaks, and 2 = hemorrhagic ulcer. Histopathological studies were also performed by preserving the colonic part in $10 \%$ formalin solution [23].

2.7. Stability Study. The stability of curcumin microsponges (F7) was carried out as per ICH guidelines in accelerated conditions. The microsponge formulation was kept at $40^{\circ} \mathrm{C} \pm$ $2^{\circ} \mathrm{C}$ and $75 \% \pm 5 \% \mathrm{RH}$ for three months. After 3 months microsponges were analyzed for physical appearance, in vitro drug release, and FTIR spectroscopy.

\section{Results and Discussion}

As stated in Section 2, microsponges were prepared by the quasi emulsion technique and subjected to various evaluation parameters.

3.1. In Vitro Characterization of Microsponges. Prepared microsponges were characterized for physical appearance, microscopic evaluation, particle size, encapsulation efficiency, percentage yield, drug loading, in vitro drug release, and pharmacodynamic activity.

3.2. Particle Size, Shape, and Surface Morphology of Curcumin Microsponges. The particle size of developed microsponges was analyzed by Zeta Sizer, which showed the average size of microsponges was within the range of $41.63 \mu \mathrm{m}$ to $57.59 \mu \mathrm{m}$. The particle size of microsponges was affected by volume of solvent and concentration of Eudragit, as increase in volume of ethanol produces less viscous solution, which resulted in smaller particle size, as exhibited by F7 $(41.63 \mu \mathrm{m}), \mathrm{F} 8$ $(45.62 \mu \mathrm{m})$, and F9 $(47.23 \mu \mathrm{m})$ (Table 3$)$.

The microsponges' formulation F7 was visualized by scanning electron microscope to assess the morphology of microsponges. SEM image revealed spherical and porous surface as shown in Figure 1.

\subsection{Determination of Encapsulation Efficiency, Percentage} Yield, and Drug Loading. Table 3 shows the values of drug loading, percentage yield, and encapsulation efficiency of different batches of curcumin loaded microsponges. The PY values range between 71.49 and $88.89 \%$. The different formulations showed EE values varied between $66.12 \%$ and $78.13 \%$. The EE was found to increase with increase in volume of ethanol that may be due to the higher solubilization of drug in ethanol. Higher volume of solvent resulted in uniform mixing of drug and solvent which resulted in higher EE. Moreover, particle size of microsponges also affected the encapsulation efficiency. EE was found to increase with decrease in the particle size. This might be due to the fact that smaller particle size will offer more surface area for adsorption of drug. F7 formulation having smallest particle size $(41.63 \mu \mathrm{m})$ showed highest EE of $78.13 \%$. 
TABLE 3: Compilation of various evaluation parameters for curcumin microsponge formulations.

\begin{tabular}{lcccc}
\hline Formulation code & $\begin{array}{c}\text { Particle size } \\
(\mu \mathrm{m})\end{array}$ & EE $(\%)^{*}$ & PY $(\%)^{*}$ & DL $(\%)^{*}$ \\
\hline F1 & $51.06 \pm 0.15$ & $70.36 \pm 1.23$ & $87.26 \pm 0.42$ & $37.71 \pm 0.22$ \\
F2 & $54.44 \pm 0.96$ & $68.75 \pm 0.98$ & $88.89 \pm 0.92$ & $40.18 \pm 0.56$ \\
F3 & $57.59 \pm 0.55$ & $66.12 \pm 1.96$ & $74.45 \pm 0.74$ & $45.36 \pm 0.74$ \\
F4 & $50.14 \pm 0.71$ & $76.61 \pm 0.84$ & $86.13 \pm 1.55$ & $69.45 \pm 0.98$ \\
F5 & $52.95 \pm 0.22$ & $74.85 \pm 0.56$ & $86.39 \pm 1.78$ & $40.33 \pm 1.20$ \\
F6 & $55.29 \pm 0.81$ & $71.19 \pm 1.10$ & $80.44 \pm 0.71$ & $64.45 \pm 0.77$ \\
F7 & $41.63 \pm 0.25$ & $78.13 \pm 1.45$ & $80.15 \pm 0.84$ & $66.94 \pm 0.68$ \\
F8 & $45.62 \pm 0.69$ & $76.74 \pm 0.73$ & $84.72 \pm 0.58$ & $47.69 \pm 1.73$ \\
F9 & $47.23 \pm 0.74$ & $72.58 \pm 2.31$ & $71.49 \pm 1.19$ & $39.89 \pm 0.84$ \\
\hline
\end{tabular}

${ }^{*} n=3$, mean \pm S.D.

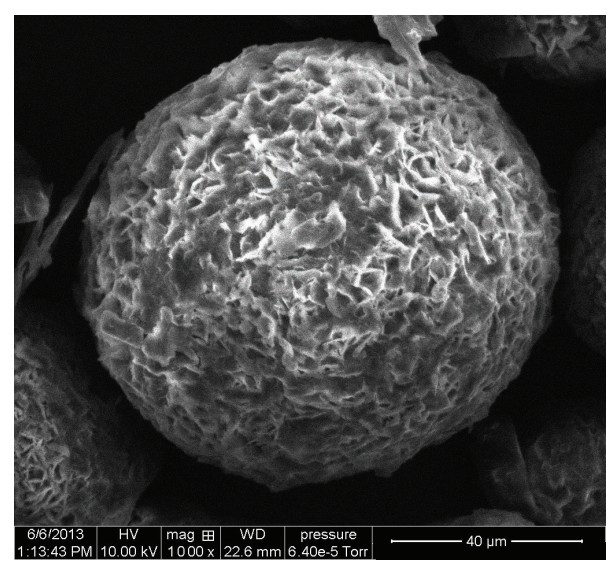

FIGURE 1: SEM photomicrograph of curcumin microsponges.

3.4. In Vitro Release Study of Curcumin Microsponges. In vitro release study of curcumin microsponges was carried out at different $\mathrm{pH}(\mathrm{pH} 1.2$ for $2 \mathrm{~h}, \mathrm{pH} 4.5$ for next $2 \mathrm{~h}$, and $\mathrm{pH} 6.8$ with $4 \% \mathrm{w} / \mathrm{v}$ fresh rat caecal content under anaerobic condition for subsequent hours) to mimic the GIT environment. The studies were carried out in $900 \mathrm{~mL}$ of the dissolution medium at $37 \pm 0.5^{\circ} \mathrm{C}$. The in vitro release of drug from microsponges is shown in Figure 2(a). Results of in vitro drug release revealed that 4.53 to $7.91 \%$ of CUR was released from the microsponges in initial $4 \mathrm{~h}$ and 51.25 to $84.12 \%$ of drug was released after $12 \mathrm{~h}$. The drug release suggested that Eudragit L100 prevented the premature release of curcumin in the upper GIT, since it is a $\mathrm{pH}$ sensitive polymer having threshold $\mathrm{pH}$ value above 6 , which bypasses the GIT and showed drug release above $\mathrm{pH}$ 6. Release rate of curcumin from microsponges increased after $4 \mathrm{~h}$, due to the exposure of formulations to $\mathrm{pH} 6$ which is above the solubilizing $\mathrm{pH}$ of Eudragit L100 polymer $[11,24]$. Eudragit L100 also increased the solubility of curcumin by $62.87 \%$. It was due to decrease in the particle size of drug to molecular level. Formulations F7, F4, and F1 exhibited higher drug release as compared to other formulations due to lower level of Eudragit. In vitro release of drug from F7 in presence and absence of RCC is depicted in Figure 2(b). Results revealed that in presence of
RCC the release of drug from the microsponges was slightly higher, which might be due to the erosion of polymeric matrix in presence of RCC.

3.5. Optimization of Experimental Design. The optimization studies were performed by using Design Expert software version 8.0.7.1 and second order polynomial equation was derived after transformation of variables (Figure 3). Further theoretical formulation F10 was designed as an extra checkpoint. The experimental value of \% cumulative drug release (CDR) was compared with predicted value and there was no significant difference between the two values with similarity factor of $92.56(P<0.05)$, thus confirming the validity of optimization. The transformed equations are as follows. Equation (2) represented equation for drug release, (3) is for particle size, and (4) is for entrapment efficiency:

$$
\begin{aligned}
Y=62.9+ & 1.45 X_{1}+11.1 X_{1}^{2}+8 X_{2}-7.51 X_{2}^{2}+1.85 X_{1} X_{2} \\
Y= & 52.8-4.51 X_{1}-2.48 X_{1}^{2}+2.86 X_{2}-0.52 X_{2}^{2} \\
& +0.30 X_{1} X_{2} \\
Y= & 68.91+4.69 X_{1}-2.8 X_{1}^{2}-2.01 X_{2}-1.65 X_{2}^{2} \\
& -1.03 X_{1} X_{2} .
\end{aligned}
$$

3.6. Selection of Optimized Formulation. F7 formulation was identified as optimized formulation based on the smallest particle size $(41.63 \pm 0.25 \mu \mathrm{m})$, the highest $\mathrm{EE}(78.13 \pm$ $1.45 \%)$, the highest drug release $(84.12 \%)$, and desirability factor of 0.83 . Further the F7 formulation was used for pharmacodynamic evaluation. The drug release profile of F7 formulation was subjected to different kinetic models and based upon the best correlation coefficient $\left(r^{2}=0.9927\right)$ the release was found to follow Higuchi model, which suggested diffusion as the main mechanism of drug release.

3.7. In Vivo Study. In vivo study using acetic acid induced colitis model in rat showed that ulcers in curcumin loaded microsponges treated group were recovered up to a good 


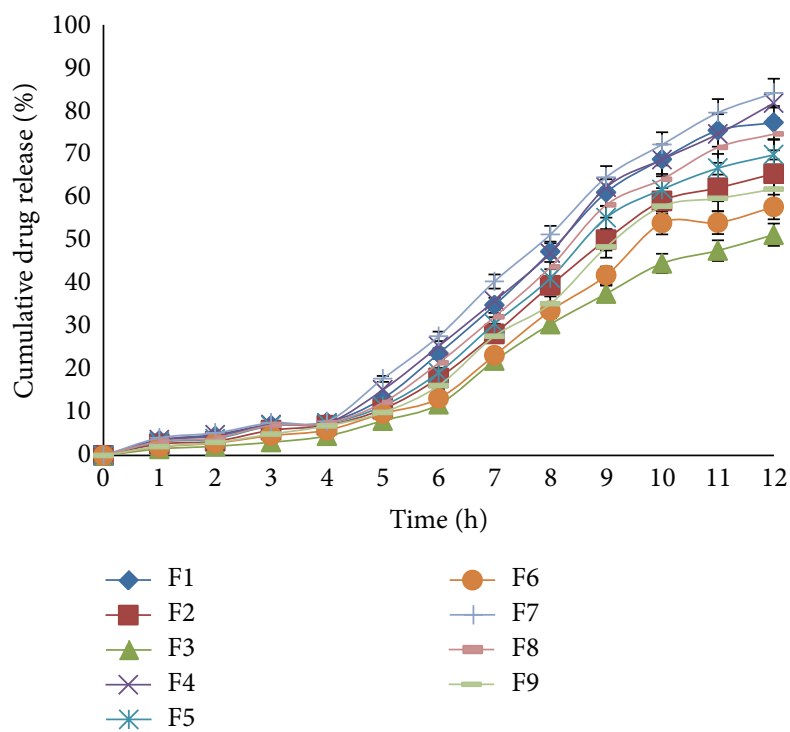

(a)

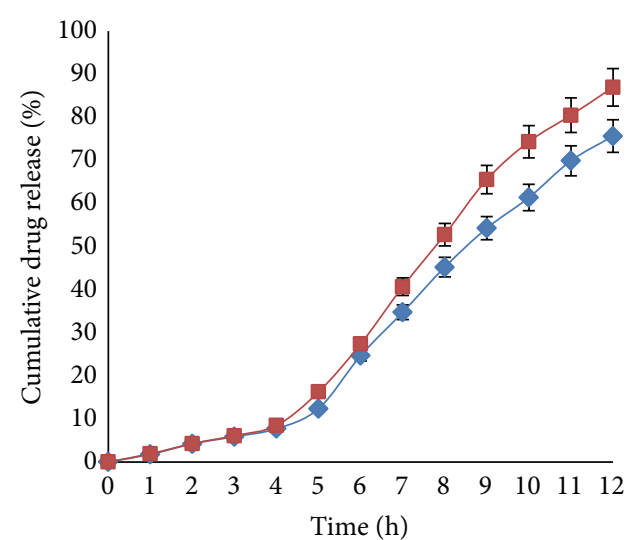

$\checkmark$ F7 (without RCC)

F7 (with RCC)

(b)

FIGURE 2: (a) Comparative dissolution profile of curcumin loaded microsponges (F1-F9). (b) In vitro release profile of F7 with and without RCC.

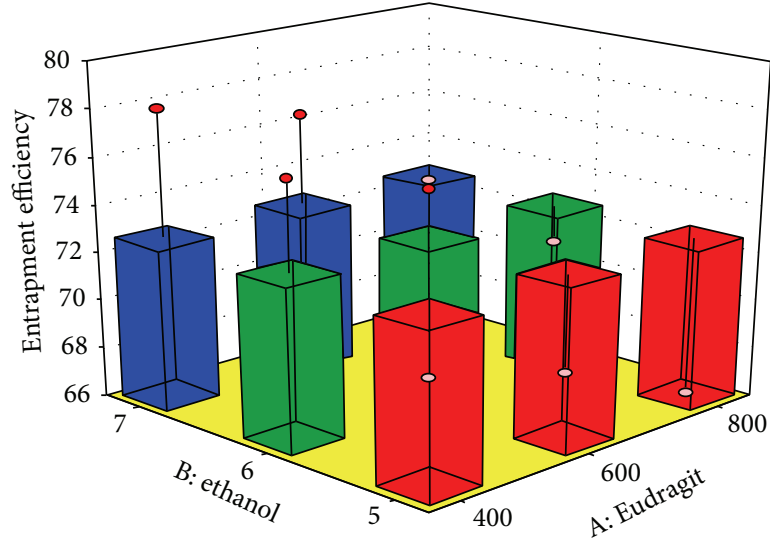

(a)

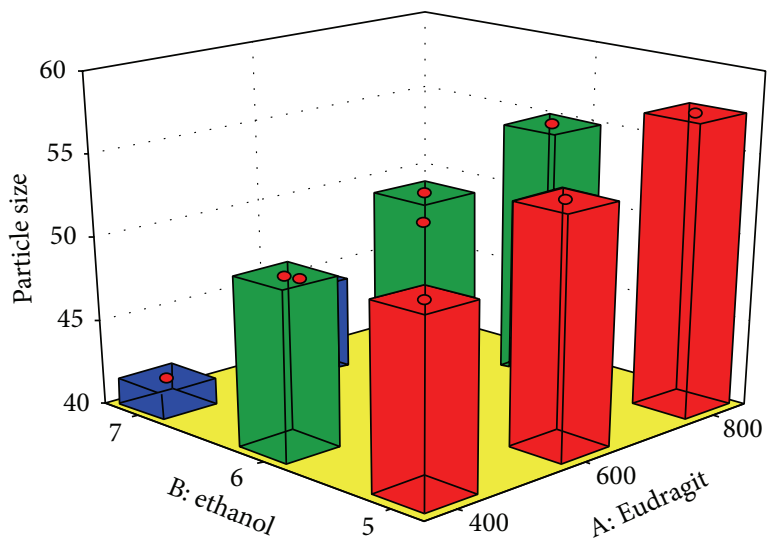

(b)

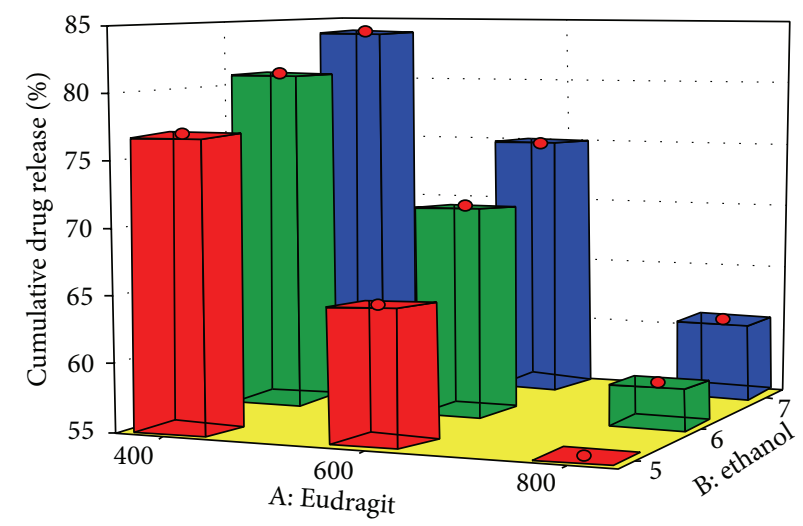

(c)

FIGURE 3: 3D bar surface chart depicting the influence of independent variables over dependent variables. 


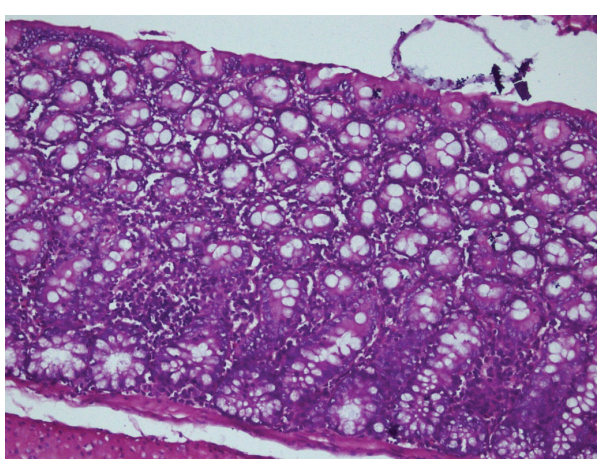

(a)

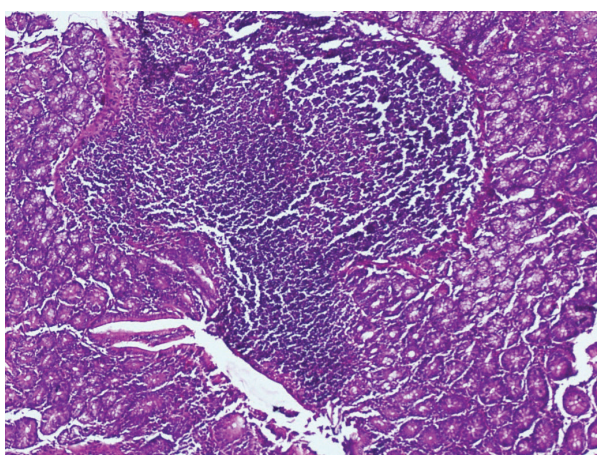

(c)

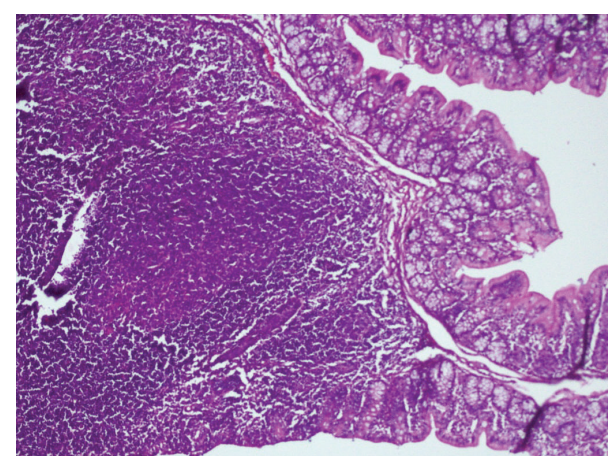

(b)

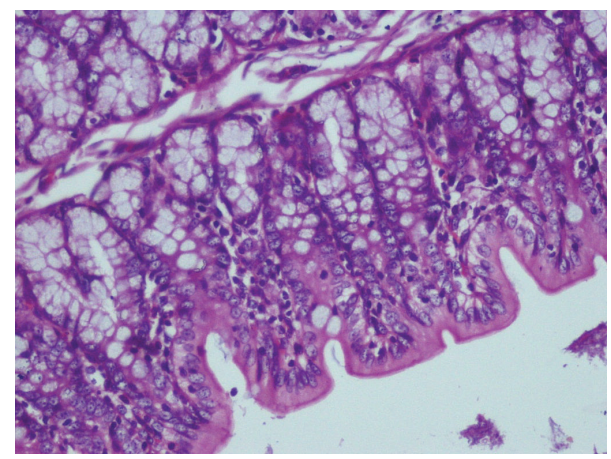

(d)

Figure 4: Histology of colonic section of (a) normal group, (b) acetic acid induced colitis group, (c) curcumin treated group, and (d) curcumin loaded microsponges treated group.

TABLE 4: Macroscopic evaluation of colonic lesions of rat.

\begin{tabular}{lccccc}
\hline \multicolumn{7}{c}{ Colonic erosion score $^{*}$} \\
\hline Groups & $\mathbf{0}$ & $\mathbf{0 . 5}$ & $\mathbf{1}$ & $\mathbf{1 . 5}$ & $\mathbf{2}$ \\
Control & - & 1 & 1 & 2 & 1 \\
Curcumin & - & 1 & 3 & 1 & - \\
Curcumin microsponges & - & 3 & 2 & - & - \\
\hline
\end{tabular}

${ }^{*} n=5$ in each group; $0=$ normal colored colon, $0.5=$ red coloration, $1=$ spot ulcer, $1.5=$ hemorrhagic streaks, and $2=$ hemorrhagic ulcer.

extent as compared to control group while in pure curcumin treated group the healing was mild. Results are shown in Table 4. Sections of colon were also assessed for histopathological studies.

3.8. Histopathology Studies. After examining the sections of the colon of the rat, it was revealed that acetic acid induced edema in submucosal layer and caused hemorrhage, necrosis, and intense inflammation on colon (Figure 4(b)). While in case of normal rat colon, there was no inflammation as shown in Figure 4(a). Curcumin treated group showed decrease in ulceration, necrosis, and hemorrhage up to a moderate extent as shown in Figure 4(c). However, the curcumin microsponges treated group showed significant decrease in these pathological parameters as compared to free curcumin (Figure $4(d)$ ). These results suggested that the microsponges

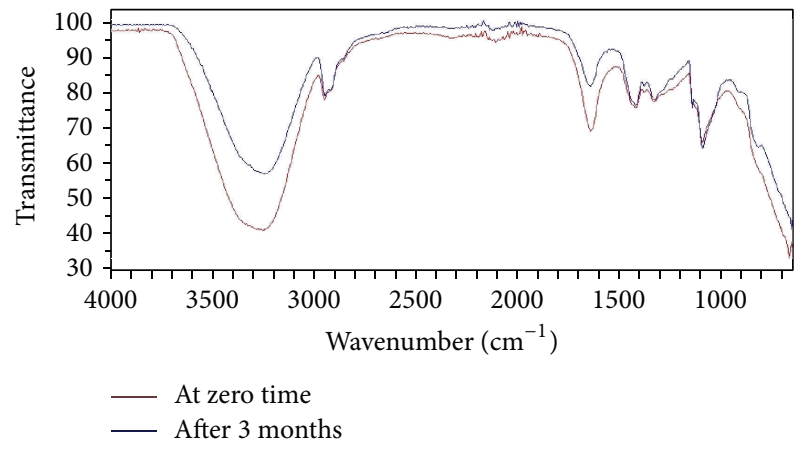

FIGURE 5: FTIR spectra depicting stability of developed formulation.

of curcumin may be used as a promising drug delivery system for treatment of ulcerative colitis.

3.9. Stability Studies. The F7 formulation was subjected to 3-month stability study at accelerated condition and was analyzed for physical appearance, in vitro drug release, and FTIR spectroscopy. After 3 months the formulation was found to show no change in physical appearance and in vitro drug release (similarity factor 91.45). The FTIR spectra revealed no sign of instability (Figure 5). Thus, all these parameters suggested that the formulation F7 may have good shelf life. 


\section{Conclusion}

Curcumin loaded microsponges were successfully developed by quasi emulsion technique for colon targeting. The developed formulation was found to be spherical with tiny pores on the surface. Eudragit L100 was used as a $\mathrm{pH}$ sensitive polymer having threshold $\mathrm{pH}$ value above 6 , which bypassed the upper GIT and showed targeted and controlled release at colonic $\mathrm{pH}$ as revealed by in vitro drug release study. The in vivo studies also revealed better therapeutic outcomes as compared to free curcumin. Thus the results suggested that curcumin loaded microsponges may be considered as a promising drug delivery system for treating ulcerative colitis.

\section{Conflict of Interests}

The authors report no declaration of interests and have received no financial support for the present work.

\section{Acknowledgment}

The authors thank Professor P. K. Khosla (vice chancellor Shoolini University) for his perennial guidance and inspiration and for providing the facilities to complete this work.

\section{References}

[1] F. Talaei, F. Atyabi, M. Azjdarzadeh, R. Dinarvand, and A. Saadatzadeh, "Overcoming therapeutic obstacles in inflammatory bowel diseases: a comprehensive review on novel drug delivery strategies," European Journal of Pharmaceutical Sciences, vol. 49, no. 4, pp. 712-722, 2013.

[2] V. Jaleh, E. Jaber, T. Naser, M. Mohsen, R. Nakisa, and D. Farid, "Development and evaluation of a novel pellet-based tablet system for potential colon delivery of budesonide," Journal of Drug Delivery, vol. 2012, Article ID 905191, 7 pages, 2012.

[3] R. Sareen, N. Jain, and K. L. Dhar, "Development of colon specific microspheres of flurbiprofen for inflammatory bowel disease," Current Drug Delivery, vol. 10, no. 5, pp. 564-671, 2013.

[4] R. Sareen, N. Jain, and K. L. Dhar, "An insight to colon targeted drug delivery system," Drug Delivery Letters, vol. 3, no. 2, pp. 127-135, 2013.

[5] R. Srivastava and K. Pathak, "Microsponges: a futuristic approach for oral drug delivery," Expert Opinion on Drug Delivery, vol. 9, no. 7, pp. 863-878, 2012.

[6] N. Amrutiya, A. Bajaj, and M. Madan, "Development of microsponges for topical delivery of mupirocin," AAPS PharmSciTech, vol. 10, no. 2, pp. 402-409, 2009.

[7] R. Sareen, N. Jain, and V. Pandit, "Curcumin: a boon to colonic diseases," Current Drug Targets, vol. 14, no. 10, pp. 1210-1218, 2013.

[8] M.-X. Ye, Y. Li, H. Yin, and J. Zhang, "Curcumin: updated molecular mechanisms and intervention targets in human lung cancer," International Journal of Molecular Sciences, vol. 13, no. 3, pp. 3959-3978, 2012.

[9] A. Karewicz, D. Bielska, B. Gzyl-Malcher, M. Kepczynski, R. Lach, and M. Nowakowska, "Interaction of curcumin with lipid monolayers and liposomal bilayers," Colloids and Surfaces B: Biointerfaces, vol. 88, no. 1, pp. 231-239, 2011.

[10] H. A. Aziz, K. K. Peh, and Y. T. F. Tan, "Solubility of core materials in aqueous polymeric solution effect on microencapsulation of curcumin," Drug Development and Industrial Pharmacy, vol. 33, no. 11, pp. 1263-1272, 2007.

[11] M. Z. I. Khan, H. P. Štedul, and N. Kurjaković, "A pH-dependent colon-targeted oral drug delivery system using methacrylic acid copolymers. II. Manipulation of drug release using Eudragit L100 and Eudragit S100 combinations," Drug Development and Industrial Pharmacy, vol. 26, no. 5, pp. 549-554, 2000.

[12] R. Srivastava, D. Kumar, and K. Pathak, "Colonic luminal surface retention of meloxicam microsponges delivered by erosion based colon-targeted matrix tablet," International Journal of Pharmaceutics, vol. 427, no. 2, pp. 153-162, 2012.

[13] L. Perge, M. Robitzer, C. Guillemot, J. Devoisselle, F. Quignard, and P. Legrand, "New solid lipid microparticles for controlled ibuprofen release: formulation and characterization study," International Journal of Pharmaceutics, vol. 422, no. 1-2, pp. 5967, 2012.

[14] R. Sareen, S. Kumar, and G. D. Gupta, "Meloxicam carbopolbased gels: characterization and evaluation," Current Drug Delivery, vol. 8, no. 4, pp. 407-415, 2011.

[15] K. S. Parvathy, P. S. Negi, and P. Srinivas, "Antioxidant, antimutagenic and antibacterial activities of curcumin- $\beta$-diglucoside," Food Chemistry, vol. 115, no. 1, pp. 265-271, 2009.

[16] S. Jose, M. T. Prema, A. J. Chacko, A. C. Thomas, and E. B. Souto, "Colon specific chitosan microspheres for chronotherapy of chronic stable angina," Colloids and Surfaces B: Biointerfaces, vol. 83, no. 2, pp. 277-283, 2011.

[17] B. Nath and L. K. Nath, "development and optimization of oral colon targeted delivery system of azathioprine using biodegradable polymers," Pharmaceutical Development and Technology, vol. 18, pp. 1131-1139, 2013.

[18] W. S. Omwancha, R. Mallipeddi, B. L. Valle, and S. H. Neau, "Chitosan as a pore former in coated beads for colon specific drug delivery of 5-ASA," International Journal of Pharmaceutics, vol. 441, no. 1-2, pp. 343-351, 2013.

[19] G. Tahan, E. Aytac, H. Aytekin et al., "Vitamin e has a dual effect of anti-inflammatory and antioxidant activities in acetic acidinduced ulcerative colitis in rats," Canadian Journal of Surgery, vol. 54, no. 5, pp. 333-338, 2011.

[20] A. D. Kandhare, K. S. Raygude, P. Ghosh et al., "Effect of hydroalcoholic extract of Hibiscus rosa sinensis Linn. leaves in experimental colitis in rats," Asian Pacific Journal of Tropical Biomedicine, vol. 2, no. 5, pp. 337-344, 2012.

[21] S. U. Gandhy, K. Kim, L. Larsen, R. J. Rosengren, and S. Safe, "Curcumin and synthetic analogs induce reactive oxygen species and decreases specificity protein (Sp) transcription factors by targeting microRNAs," BMC Cancer, vol. 12, article 564, 2012.

[22] A. Cetinkaya, E. Bulbuloglu, E. B. Kurutas, H. Ciralik, B. Kantarceken, and M. A. Buyukbese, "Beneficial effects of Nacetylcysteine on acetic acid-induced colitis in rats," The Tohoku Journal of Experimental Medicine, vol. 206, no. 2, pp. 131-139, 2005.

[23] M. Barollo, V. Medici, R. D’Incà et al., “Antioxidative potential of a combined therapy of anti $\mathrm{TNF} \alpha$ and $\mathrm{Zn}$ acetate in experimental colitis," World Journal of Gastroenterology, vol. 17, no. 36, pp. 4099-4103, 2011.

[24] D. G. Yu, Y. Xu, Z. Li, L. P. Du, B. G. Zhao, and X. Wang, "Coaxial electrospinning with mixed solvents: from flat to round eudragit L100 nanofibers for better colon-targeted sustained drug release profiles," Journal of Nanomaterials, vol. 2014, Article ID 967295, 8 pages, 2014. 

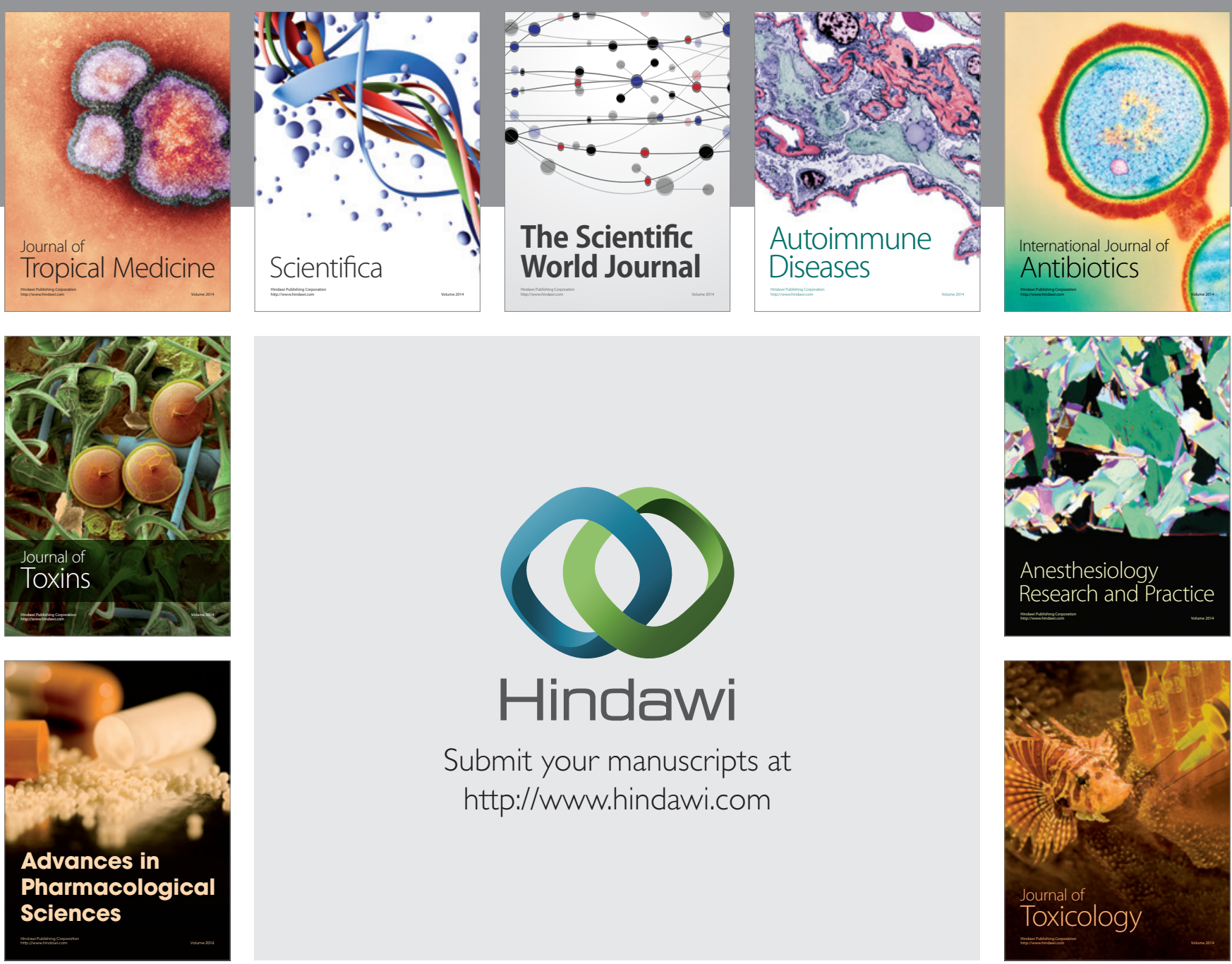

\section{Hindawi}

Submit your manuscripts at

http://www.hindawi.com
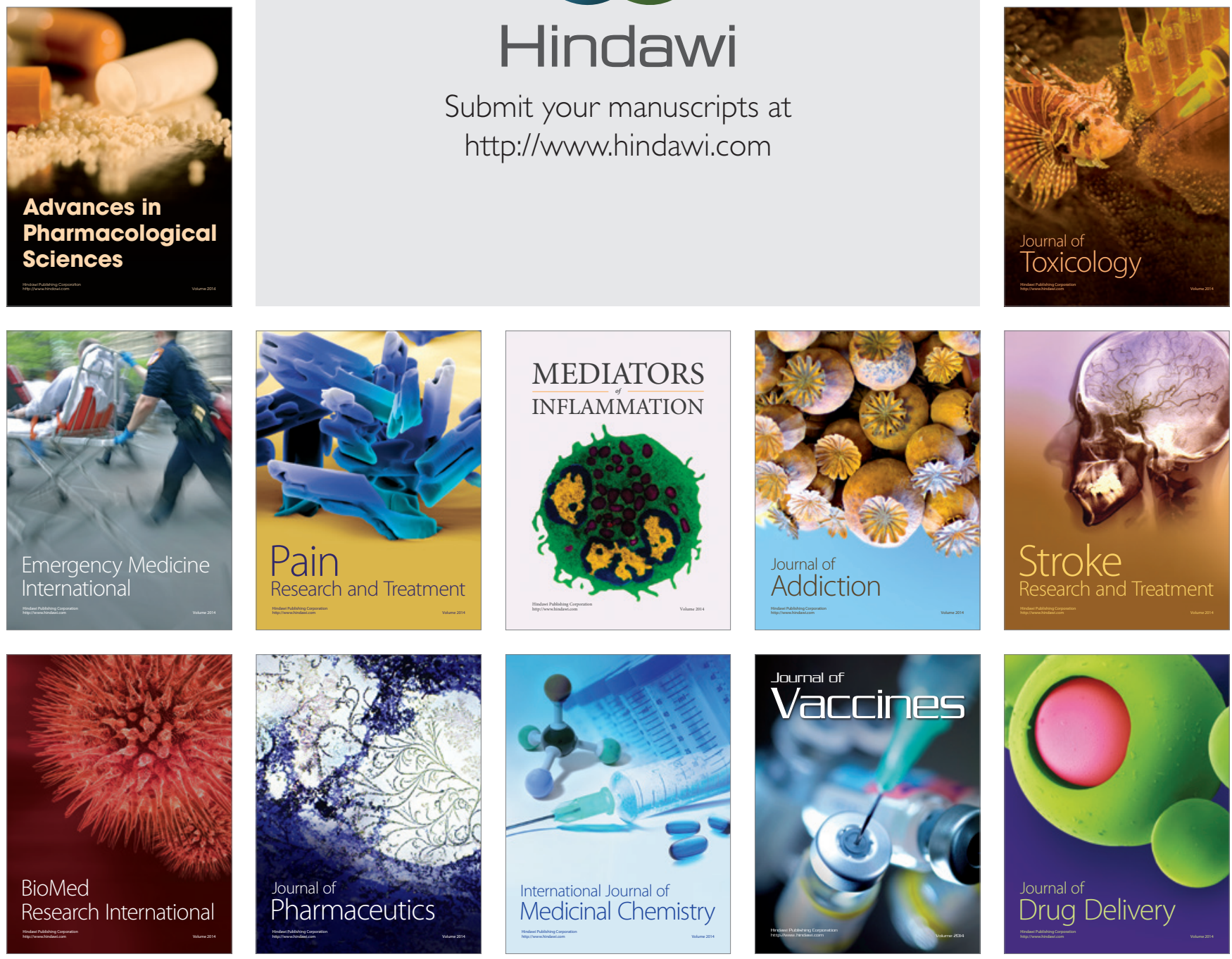